\title{
Effects of short-pulsed laser radiation on transient heating of superficial human tissues
}

\author{
Jaona H. Randrianalisoa ${ }^{a}$, Leonid A. Dombrovsky ${ }^{\text {b,* }}$, Wojciech Lipiński ${ }^{c}$, Victoria Timchenko ${ }^{\mathrm{d}}$ \\ ${ }^{a}$ GRESPI, University of Reims Champagne-Ardenne, EA 4694, Moulin de la Housse, F-51687, Reims, France \\ b Joint Institute for High Temperatures, Krasnokazarmennaya 17A, NCHMT, 111116, Moscow, Russia \\ ${ }^{\mathrm{c}}$ Research School of Engineering, The Australian National University, Canberra ACT 0200, Australia \\ ${ }^{\mathrm{d}}$ School of Mechanical and Manufacturing Engineering, University of New South Wales, Sydney 2052, Australia
}

\section{A R T I C L E I N F O}

\section{Article history:}

Received 29 May 2014

Received in revised form 6 July 2014

Accepted 7 July 2014

Available online 27 July 2014

\section{Keywords:}

Radiative transfer

Transient heat transfer

Bio-medical applications

Hyperthermia

Computational methods

Pulsed laser

Unsteady effects

\begin{abstract}
A B S T R A C T
Transient radiative transfer effects are pertinent to thermal treatment of superficial cancer via shortpulsed laser irradiation. Importance of the transient effects arise from relatively strong scattering and long attenuation path of radiation in human tissues in the therapeutic window until the complete absorption. Our analysis is based on transport approximation for scattering phase function and the Monte Carlo method for solving the three-dimensional radiative transfer problem. Monte Carlo simulations are used to study applicability of the quasi-steady radiative transfer approach, and demonstrate that in the biomedical problem under consideration, the quasi-steady solution for absorbed radiation power is sufficiently accurate for laser pulse duration longer than $10 \mathrm{ps}$. The simulations for superficial tissues with embedded gold nanoshells, used to increase the local volumetric absorption, show that overheating of the nanoshells with respect to the ambient biological tissue is strongly dependent on the laser pulse duration. This effect becomes considerable for laser pulse duration shorter than 1-2 ns. The quasi-steady approach for radiative transfer results in significantly underestimated temperatures of human tissues for short laser radiation pulses. The latter is explained by a relatively strong reflection of the short pulsed radiation by highly scattering human tissues.
\end{abstract}

(c) 2014 Elsevier Ltd. All rights reserved.

\section{Introduction}

Thermal treatment of superficial cancer via short-pulsed laser radiation has been extensively studied in the last decade [1-3]. Visible and near-infrared laser radiation in the spectral range corresponding to the so-called therapeutic window, typically $0.6-$ $1.4 \mu \mathrm{m}[4,5]$, is used for tumor heating in the direct (DHA) [6-8] and indirect (IHA) $[9,10]$ heating approaches. In IHA, an annular region around the tumor is irradiated and the tumor is heated predominantly by conduction during the time intervals between periodic laser irradiation. In both approaches, heating can be enhanced by embedding strongly absorbing gold nanoparticles (GNPs) [8,11-14]. IHA can also perform well without the use of GNPs $[9,10]$, alleviating the difficulties associated with nanoparticle supply and concentration control.

An accurate computational model for thermal treatment of superficial tumors, e.g. such as melanoma, by IHA must capture the relevant bio-heat transfer effects such as the metabolic heat

\footnotetext{
* Corresponding author.

E-mail address: ldombr@yandex.ru (L.A. Dombrovsky).
}

generation, thermal non-equilibrium between arterial blood and ambient tissues and kinetics of the cell damage, as well as absorption of laser radiation in anisotropically scattering multi-layer human tissues. An approximate radiative transfer model with application to IHA [15] was examined by the authors in [16]. The transport approximation for the scattering phase function and differential models for radiative transfer $[17,18]$, in particular the modified two-flux approximation [19], were shown to be sufficiently accurate at an acceptable computational cost. The study presented in [16] was based on a steady-state approach to radiative transfer, thus neglecting possible unsteady effects that may arise when very short pulses of the incident laser radiation are used.

Heat transfer in human tissues without GNPs or with GNPs remaining in thermal equilibrium with the ambient tissues can be analyzed using the radiation power absorbed in the tissues during a relatively long time as compared with the pulse duration. However, for highly absorbing GNPs embedded in the tissue the thermal equilibrium condition may not be satisfied for short laser pulses which might result in microscale thermal effects around GNPs. Consequently, knowledge of a transient radiative intensity 


\begin{tabular}{|c|c|c|c|}
\hline \multicolumn{4}{|c|}{ Nomenclature } \\
\hline$a$ & particle radius, $\mathrm{m}$ & $\tau_{\mathrm{m}}$ & averaging time period, s \\
\hline$A_{\text {inc }}$ & irradiated surface area, $\mathrm{m}^{2}$ & $\Psi$ & probability of absorption \\
\hline$c_{0}$ & speed of light in vacuum, $\mathrm{m} \mathrm{s}^{-1}$ & & \\
\hline$d$ & thickness, m & \multicolumn{2}{|c|}{ Subscripts and superscripts } \\
\hline$d_{\mathrm{pp}}$ & particle-particle separation distance, m & a & absorbed \\
\hline$f_{\mathrm{v}}$ & volume fraction of particles & $\mathrm{C}$ & cooling, central \\
\hline$m_{\mathrm{s}}$ & number of scattering events & e & extinction, external \\
\hline$M$ & number of pulses & $\mathrm{g}$ & gold \\
\hline$n$ & real part of complex refractive index & i & initial \\
\hline$N$ & ray number & Inc. & incident \\
\hline$P$ & energy per unit area, $\mathrm{J} \mathrm{m}^{-2}$ & $\max$ & pulse maxima \\
\hline$q$ & irradiation, $\mathrm{W} \mathrm{m} \mathrm{m}^{-2}$ & $\mathrm{n}$ & normal to a surface \\
\hline Q & efficiency factor & $\mathrm{p}$ & particle, pulse \\
\hline$r, r^{\prime}$ & radius & qS & quasi-steady \\
\hline$R$ & random number & ray & ray number \\
\hline$S_{\mathrm{e}}$ & free path of a ray until extinction & S & scattered \\
\hline$t_{\mathrm{p}}$ & pulse width, s & sat & saturation \\
\hline$w$ & energy (or rate) of ray bundles, J (or W) & si & silica \\
\hline W & volumetric absorbed power density, $\mathrm{W} \mathrm{m}^{-3}$ & $\mathrm{t}$ & tissue, time, transmitted \\
\hline & & $\operatorname{tr}$ & transport \\
\hline Gree & nbols & trans & transmission \\
\hline$\alpha$ & absorption coefficient, $\mathrm{m}^{-1}$ & $\lambda$ & spectral \\
\hline$\delta$ & relative radius of the silica core & $*$ & normalized \\
\hline
\end{tabular}

field is needed to determine the thermal conditions at the microscale. The micro- and macroscale heat transfer problems are coupled because of possible thermal damages of biological cells in the vicinity of relatively hot nanoparticles. By neglecting any local thermal damage of bio-tissues, the solutions to micro- and macroscale heat transfer problems can be decoupled. A comparison of the speed of light and the speed of sound in human tissues demonstrates that the transfer of thermal energy at the finite speed needs to be taken into account for certain conditions therefore necessitating application of a modified heat conduction equation in both the micro- and macroscale thermal analysis with appropriate relaxation times matching the different characteristic time scales. Previous pertinent studies of non-Fourier conduction in presence of radiative transfer can be found in the references [20-22]. Note that the use of the Cattaneo's hyperbolic heat conduction model may lead to unphysical results for very short time scales or very small dimensions, for which the local thermal equilibrium assumption is not valid [23,24]. It was shown in the recent study [25] that the dual-phase-lag model of heat conduction proposed by Tzou [26-28] also provides incorrect predictions in some cases. The non-Fourier conduction problem is closely related to the problem under consideration in the present study. At the same time, a non-Fourier analysis of heat transfer processes in the vicinity of GNPs is beyond the scope of the present study.

The problem of a transient radiative transfer has been addressed in numerous investigations, especially in the last decade [29-49]. However, only few of these studies were concerned with biomedical applications. Note that the problem statements in some recent papers [42,46-49] are close to that in the present paper. At the same time, to the best of our knowledge, there are no theoretical and computational studies in the literature concerning the problems related to interaction of short-pulsed radiation with human tissues containing embedded GNPs. It is also important that effects of a short-pulsed laser radiation on transient heating of superficial human tissues are analyzed in the present paper.

In this work, transient effects of radiative transfer in superficial human tissues during short-pulsed laser cancer treatment via IHA are considered in the case when the effects of the finite speed of light become important. Specifically, the objectives of this paper are: (1) to elucidate transient effects of radiative transfer in superficial human tissues including the case with embedded highlyabsorbing GNPs subject to short-pulsed laser irradiation, and (2) to identify the limiting laser pulse duration for which the quasisteady approach to radiative transfer in superficial human tissues remains valid.

To estimate the macroscale effects of the short-pulsed irradiation on the temperature field in multilayer superficial human tissues, the transient heat transfer problem at realistic conditions of hyperthermia $[9,10]$ is solved for both continuous and short-pulsed irradiation.

\section{Problem statement}

A schematic of an axisymmetric superficial human tissue section as applied to the thermal cancer treatment via IHA is shown in Fig. 1. The section includes five zones corresponding to different layers of human tissue (Fig. 1a), namely epidermis (\#1), papillary and reticular dermis (\#2), fat (\#3), muscle (\#4), and superficial tumor (\#5). The transient light propagation in absorbing and scattering human tissues is modeled using a 3-D radiative transfer model. A 2-D view of the axisymmetric computational domain is shown in Fig. 1b. Since the external laser radiation does not typically reach the muscle layer even in the therapeutic window [9], this layer is not included in the actual computational domain shown in Fig. 1b. The tumor is not directly irradiated in IHA, and thus it is also excluded from the computational domain shown in Fig. 1b. Both zones should be included in their entirety in heat transfer models of superficial tissues undergoing long-time thermal treatment $[9,10,15]$.

An annular region of the body surface $A_{\text {inc }}$ surrounding the superficial tumor is exposed to short-pulsed laser irradiation. The irradiated area of the body surface is flat and optically smooth. This assumption is justified by a predominant contribution of 

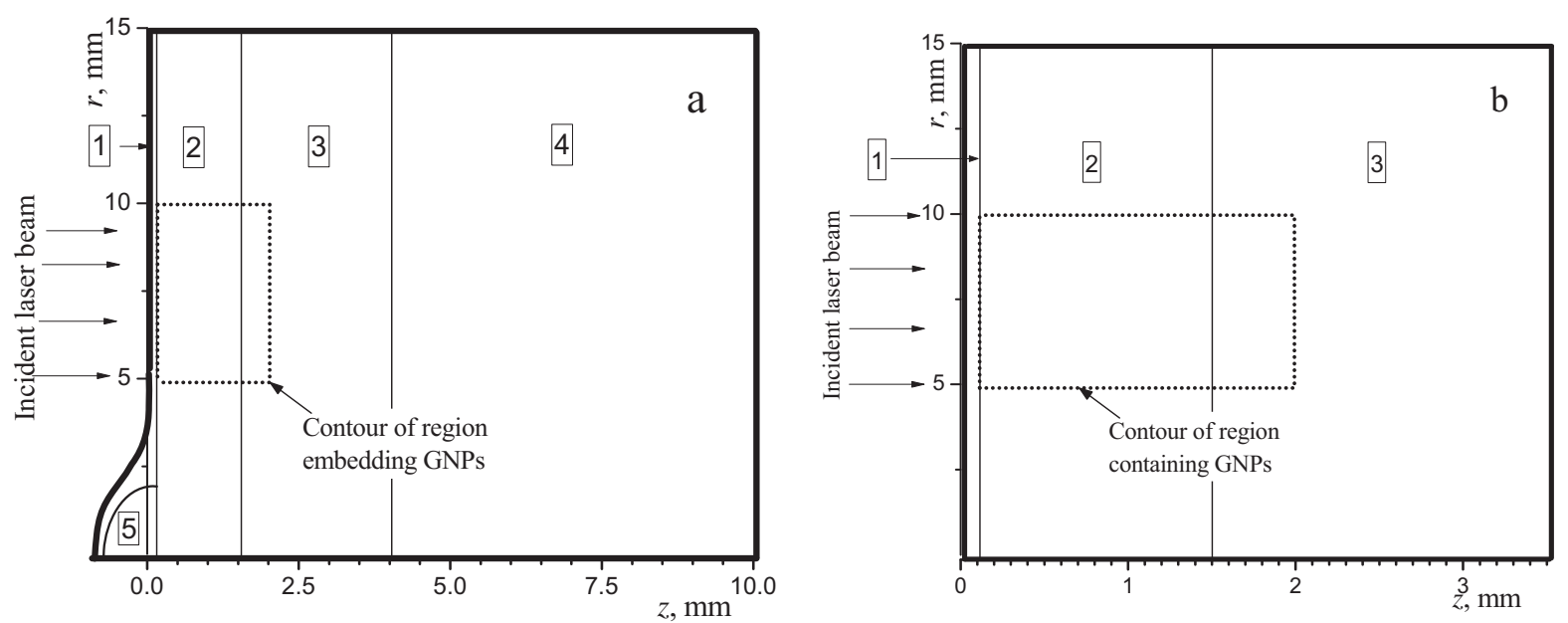

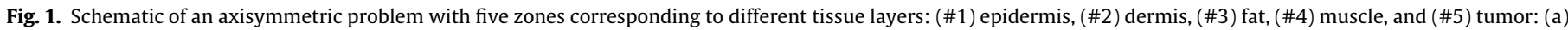
2-D tissue section including the central (non-irradiated) and annular irradiated regions; and (b) 2-D view of the computational domain.

volumetric scattering of radiation in human tissues to the reflectance. Two variants are considered: (i) IHA with gold nanoparticles consisting of a silica core enclosed in a gold shell, referred to as gold nanoparticles (GNPs) in the following text, embedded in an annular region $\left(r_{1} \leqslant r \leqslant r_{2}, \quad r_{1}=5 \mathrm{~mm}, \quad r_{2}=10 \mathrm{~mm}\right.$ and $0.1 \leqslant z_{1} \leqslant 2 \mathrm{~mm}$ ) including parts of the dermis and fat layers, which in turn are located underneath the plane of incidence of the laser beam, and (ii) IHA without GNPs.

For simplicity, it is assumed that for the variant with GNPs, their volume fraction is uniform in the suspension. Although the simulations are performed in the entire domain depicted in Fig. 1b, the absorbed radiative power is evaluated only for a cylindrical sub-domain of $3.5-\mathrm{mm}$ thickness, $2.5-\mathrm{mm}$ internal radius and $12.5-\mathrm{mm}$ external radius, which includes parts of zones (\#1)(\#3). The relatively small thickness of $3.5 \mathrm{~mm}$ is chosen based on the findings presented in [16] to elucidate the transient radiative transfer effects in the region of considerable absorption of laser radiation at wavelength of $0.6328 \mu \mathrm{m}$.

Separate computations are performed for a single pulse with Gaussian temporal shape and a train of Gaussian pulses given by [49]:

$q_{\mathrm{e}}(t)=q_{\mathrm{e}, \max } \sum_{i=1}^{M} \exp \left[-4 \times \ln 2 \times\left(\frac{t-t_{\mathrm{c}}-(i-1) T_{\mathrm{p}}}{t_{\mathrm{p}}}\right)^{2}\right]$

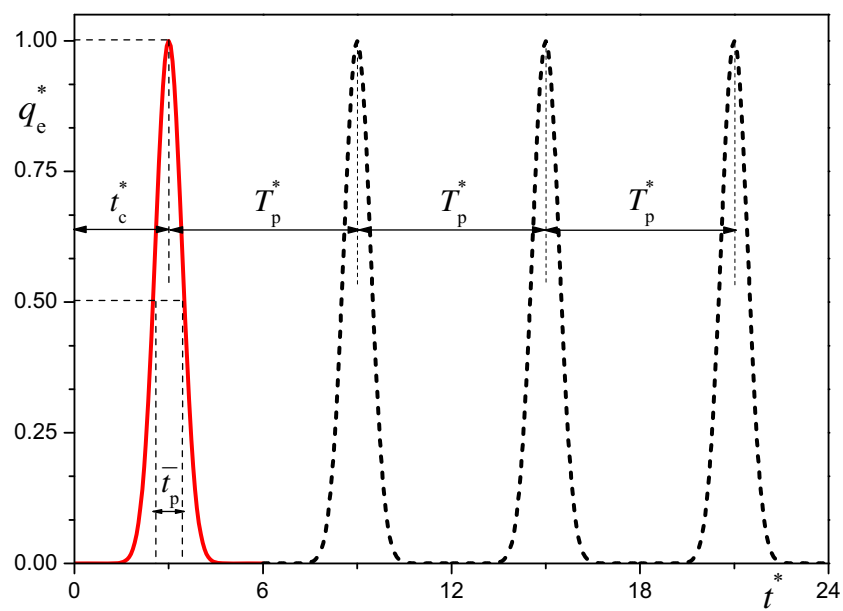

Fig. 2. The shape of a single pulse (solid curve) and a train of four pulses. where $M$ is the number of pulses. A pulse is characterized by the pulse maximum $q_{\mathrm{e}, \mathrm{max}}$, the width at half maximum $t_{\mathrm{p}}$, the time-shift of the first pulse maximum taken equal to $t_{\mathrm{c}}=1.5 t_{\mathrm{p}}$, and, in the case of periodic pulse in accordance with parameters used in [49], the pulse period $T_{\mathrm{p}}=6 t_{\mathrm{p}}$. The Gaussian pulses $q_{\mathrm{e}}^{*}\left(t^{*}\right)=q_{\mathrm{e}}\left(t^{*}\right) / q_{\mathrm{e} \text {, max }}$ are plotted in Fig. 2 as a function of the relative time $t^{*}=t / t_{\mathrm{p}}$.

The tissue index of refraction at $\lambda=0.6328 \mu \mathrm{m}$ is assumed to be uniform in the computational region, $n_{\mathrm{t}}=1.45$, even though its spatial variation in real multi-layered tissues has been reported in the range 1.341 .45 [49]. The directional-hemispherical reflectivity of the tissue surface is obtained using Fresnel's equation for an interface between two non-absorbing media:

$\rho=\frac{1}{2} \frac{\sin ^{2}\left(\theta_{\text {inc }}-\theta_{\text {trans }}\right)}{\sin ^{2}\left(\theta_{\text {inc }}+\theta_{\text {trans }}\right)}\left[1+\frac{\cos ^{2}\left(\theta_{\text {inc }}+\theta_{\text {trans }}\right)}{\cos ^{2}\left(\theta_{\text {inc }}-\theta_{\text {trans }}\right)}\right] \sin \theta_{\text {trans }}=n_{\mathrm{t}} \sin \theta_{\text {inc }}$

where $\theta_{\text {inc }}$ and $\theta_{\mathrm{t}}$ are the angles of incidence and transmission, respectively [50].

Radiative properties of spherical GNPs are predicted using Mie theory [51], while the spectral optical properties of biological tissues at $\lambda=0.6328 \mu \mathrm{m}$ are retrieved from experimental studies reported in the literature $[4,5,52]$. The effects of finite light propagation time over distances comparable with the size of GNPs are not considered. Applicability of this approach for GNPs of radius $a$ is examined using the relation

$t_{\mathrm{p}}=2 a n_{\mathrm{t}} / c_{0}$

where $c_{0}$ is the speed of light in vacuum. For $a=20 \mathrm{~nm}$, we obtain $t_{\mathrm{p}} \approx 2 \times 10^{-4} \mathrm{ps}$. The time of light propagation for a distance equal to the wavelength, $t_{\lambda}=\lambda / c_{0}$, is about $0.03 \mathrm{ps}$ at $\lambda=1 \mu \mathrm{m}$ in vacuum. As a result, one can consider the classical Mie scattering problem when the incident radiation is a plane electromagnetic wave with nearly-constant intensity at distances much greater than the particle size. It is also known that organelles inside human cells are responsible for the scattering properties of human tissues in the therapeutic window [4]. Application of Eq. (3) to organelles of size equal to the laser wavelength confirms that the scattering properties of human tissues are not affected by laser pulses for pulses longer than approximately $0.01 \mathrm{ps}$. Consequently, typical values of optical properties of human tissues reported in the literature are employed in the present analysis.

The effects of extreme heating rates of GNPs are neglected in this study, and it is assumed that the temperature of GNPs, $T_{\mathrm{p}}$, does 
not exceed a limiting value for explosive evaporation of water in the tissue surrounding the particle. According to [53], the maximum temperature excess for overheating of pure water at atmospheric pressure is equal to $\Delta T_{\max }=T_{\mathrm{p}}-T_{\text {sat }}=320 \mathrm{~K}$, where $T_{\text {sat }}=373 \mathrm{~K}$ is the water saturation temperature. The resulting formation of steam blankets around the GNP changes dramatically its radiative properties, and significantly affects heat rates from GNPs to host tissue [17,54].

The absorption and transport scattering coefficients of a tissue with embedded gold nanoshells of radius $a$ and volume fraction $f_{\mathrm{v}}$ are calculated as follows [17]:

$\alpha=\alpha_{\mathrm{t}}+\alpha_{\mathrm{p}} \quad \sigma_{\mathrm{tr}}=\sigma_{\mathrm{tr}, \mathrm{t}}+\sigma_{\mathrm{tr}, \mathrm{p}}$

$\alpha_{\mathrm{p}}=0.75 f_{\mathrm{v}} \frac{Q_{\mathrm{a}}}{a} \quad \sigma_{\mathrm{tr}, \mathrm{p}}=0.75 f_{\mathrm{v}} \frac{Q_{\mathrm{s}}^{\mathrm{tr}}}{a}$

where $Q_{\mathrm{a}}$ and $Q_{\mathrm{s}}^{\mathrm{tr}}$ are the absorption efficiency factor and the transport scattering efficiency factor, respectively [50]. According to [15], the following parameters of GNPs are used in the present study:

$a=20 \mathrm{~nm} \quad \delta=0.725 \quad Q_{\mathrm{a}}=7.828 \quad Q_{\mathrm{s}}^{\mathrm{tr}}=1.144$

where $\delta$ is the relative radius of the silica core.

\section{Quasi-steady approach}

\subsection{Solution based on modified two-flux approximation}

The quasi-steady radiative transfer model for non-uniform human tissues with embedded nanoparticles is presented in [15], and the mathematical details are omitted from this text for brevity. For simplicity, the transport approximation is applied to the anisotropic scattering of light inside human tissue $[17,18]$. The mathematical problem is linear with respect to the incident radiative flux. Consequently the solution is obtained for the unit incident radiative flux.

\subsection{Monte Carlo ray-tracing solution}

The Monte Carlo ray-tracing (MCRT) method for quasi-steady radiative transfer modeling is well-covered in the literature, e.g. [55-57]. Thus, only the aspects specific to the present study are discussed here. The collision-based Monte Carlo method with the transport approximation to the scattering phase function is applied $[16,58]$. The simulations are carried out for the total number of rays $N_{\text {ray, }}$, with radiative power of each ray given by:

$w_{\mathrm{q}-\mathrm{s}}=A_{\text {inc }} \bar{q}_{\text {inc }} / N_{\text {ray }}$

where $\bar{q}_{\text {inc }}$ is the time-averaged irradiation flux by the laser, obtained as

$\bar{q}_{\text {inc }}=P(\tau) / \tau \quad P(\tau)=\int_{0}^{\tau} q_{\text {inc }}(t) d t$

where $\tau$ is the laser pulse duration, $P(\tau)$ is the radiative energy per unit area of the laser pulse of total duration $\tau=6 t_{\mathrm{p}}$ for a single pulse and $\tau=6 M t_{\mathrm{p}}$ for a train of $M$ pulses. During tracing, rays undergo absorption and scattering in the medium, and boundary reflection and refraction. Rays are traced until they are absorbed inside the domain, exit the domain through the tissue surface, or propagate sufficiently far from the evaluation sub-domain discussed in the previous section. Rays incident at the tissue surface from within the medium are either transmitted or internally reflected, with the choice based on the actual value of the directional-hemispherical reflectivity given by Eq. (2). Rays propagating at large distances, here assumed at $r>15 \mathrm{~mm}$ and/or $z>3.5 \mathrm{~mm}$, are considered as lost because of a low probability to be absorbed within the computational sub-domain.
The counters of absorbed rays are stored on a structured 2-D cylindrical mesh consisting of $n_{z} \times n_{r}=50 \times 20$ annular cells with the following non-uniform spatial resolution:

$\Delta z=\left\{\begin{array}{cl}0.01 \mathrm{~mm} & \text { for } 0.0 \leqslant z<0.1 \mathrm{~mm} \\ 0.07 \mathrm{~mm} & \text { for } 0.1 \leqslant z \leqslant 1.5 \mathrm{~mm} \\ 0.10 \mathrm{~mm} & \text { for } 1.5 \leqslant z \leqslant 3.5 \mathrm{~mm}\end{array} \quad \Delta r=0.5 \mathrm{~mm}\right.$

After tracing all $N_{\text {ray }}$ rays, the normalized spatial distribution of the absorbed radiative power is obtained using [16]:

$W^{*}(r, z) \approx\left(1-\rho_{\mathrm{n}}\right) \frac{w_{\mathrm{q}-\mathrm{s}}}{\bar{q}_{\mathrm{e}}} \frac{N_{\mathrm{a}}\left(i_{z}, i_{r}\right)}{V\left(i_{z}, i_{r}\right)}$,

$V\left(i_{r}, i_{z}\right)=\frac{\pi \Delta z \Delta r^{2}}{4}\left[\left(i_{r}+1\right)^{2}-i_{r}^{2}\right] \quad \rho_{\mathrm{n}}=\frac{\left(n_{\mathrm{t}}-1\right)^{2}}{\left(n_{\mathrm{t}}+1\right)^{2}}$

where $V\left(i_{r}, i_{z}\right)$ and $N_{\mathrm{a}}\left(i_{r}, i_{z}\right)$ are the volume of the cell $\left(i_{r}, i_{z}\right)$ and number of rays absorbed in this cell, respectively.

\section{Transient approach}

The transient MCRT method is selected as the method of solution for the unsteady radiative transfer problem in the nonuniform tissue to overcome numerical difficulties of solution based on the modified two-flux approximation.

\subsection{Transient Monte Carlo algorithm}

The Monte Carlo simulation of the unsteady problem is similar to the quasi-steady method described in Section 3. The main difference is that for unsteady problems, the histories of rays are evaluated at time instants and the time variation of radiative sources is taken into account during ray histories. In the unsteady approach, each ray carry energy $w$ defined by,

$w=A_{\text {inc }} P(\tau) / N_{\text {ray }}$

For a pulsed radiation (Fig. 2), the initial time, $t_{i}$, of the ray $i$ for $i=1$ to $N_{\text {ray }}$, is randomly sampled from the normalized Gaussian distribution:

$R_{\mathrm{t}}=\int_{0}^{t_{\mathrm{i}}} q_{\text {inc }}(t) \mathrm{d} t / \int_{0}^{\tau} q_{\text {inc }}(t) \mathrm{d} t=\frac{P\left(t_{i}\right)}{P(\tau)}$

with $P(\tau)$ already defined by Eq. (7).

During their paths, the rays may undergo volumetric absorption and scattering as well as boundary reflection and refraction. The determination of location and type of extinction and boundary interaction are managed in the same manner as for quasi-steady algorithm. At each extinction point, the time of flight of the ray is updated by adding the duration of the last path propagation before interaction $s_{\mathrm{e}}$ :

$t_{\mathrm{e}}=\frac{1}{c_{0}} \int_{0}^{s_{\mathrm{e}}} n_{\mathrm{t}} d s$

where $n_{\mathrm{t}}$ is the local refractive index of tissue. Consequently, the time at which the absorption of a ray $i$ occurs is given by:

$t=t_{\mathrm{i}}+\sum_{j=1}^{m_{\mathrm{s}}+1} t_{\mathrm{e}, \mathrm{j}}$

The summation in Eq. (13) is performed over the number of scattering events $m_{\mathrm{s}}$ that the ray has undergone along its path before absorption.

At each absorption event, the number of absorbed rays at time $t$ and space coordinates $z$ and $r$ is updated. In the computer code, the number of absorbed rays is stored in an array $N_{\mathrm{a}}\left(i_{t}, i_{r}, i_{z}\right)$, where $i_{t}=-$ $\operatorname{int}(t / \Delta t)+1, i_{r}=\operatorname{int}(r / \Delta r)+1$ and $i_{z}=\operatorname{int}(z / \Delta z)+1$ are the time and 
spatial indices, respectively. The absorbed power is calculated at $n_{t}=100$ equidistant time points, with the interval $\Delta t$. The spatial discretization of the computational domain is the same as in the quasi-steady approach of Section 3.

\subsection{Transient absorbed power}

In the case with embedded GNPs, the thermal equilibrium between the GNPs and the ambient tissue is normally assumed in the macroscopic thermal model. This assumption is generally applicable for the irradiation by a continuous wave (cw) laser but it may be incorrect for a short-pulsed laser. Under the thermal equilibrium assumption, the absorbed power is determined from the number of rays absorbed in each computation cell without distinction of absorbing components (GNP or tissue). Indeed, after all $N_{\text {ray }}$ rays have been traced, the volumetric distribution of the transient absorbed power and the corresponding normalized quantity are approximated as:

$W(t, r, z) \approx\left(1-\rho_{\mathrm{n}}\right) w \frac{N_{a}\left(i_{t}, i_{r}, i_{z}\right)}{\Delta t V\left(i_{r}, i_{z}\right)} \quad W^{*}(t, r, z)=\tau W(t, r, z) / P(\tau)$

The above assumption of thermal equilibrium between GNPs and ambient human tissue is applicable in the case of a relatively slow heating of the nanoparticles. At the same time cooling of small particles by thermal conduction to the surrounding medium is rapid. The cooling time can be estimated using the solution for transient cooling of a homogeneous isothermal sphere with initial temperature $T_{\mathrm{p}, 0}$ placed in an infinite host medium at temperature $T_{\mathrm{t}}$ :

$\frac{T_{\mathrm{p}}-T_{\mathrm{t}}}{T_{\mathrm{p}, 0}-T_{\mathrm{t}}}=\exp (-\mathrm{Fo}) \quad$ Fo $=\frac{k_{\mathrm{t}} t}{(\rho c)_{\mathrm{p}} a^{2}}$

where $k_{\mathrm{t}}$ is the thermal conductivity of the host medium and $(\rho c)_{\mathrm{p}}$ is the specific volumetric heat capacity of the particle substance. The size of gold nanoshells is usually much smaller than the wavelength, referred to as the Rayleigh limit [17]. Consequently, the volumetric distribution of the absorbed radiation power in a particle is uniform, justifying the isothermal approximation. The Fourier number Fo is the only dimensionless parameter that determines the particle cooling rate. A condition of Fo $=1$ provides an estimate for the cooling time:

$t_{\mathrm{c}}=(\rho c)_{\mathrm{p}} a^{2} / k_{\mathrm{t}}$

In the case of a gold nanoshell with a silica core, one can use the following relation:

$(\rho c)_{\mathrm{p}}=(\rho c)_{\mathrm{si}} \delta^{3}+(\rho c)_{\mathrm{g}}\left(1-\delta^{3}\right)$

Substituting the values of $(\rho c)_{s i}=1.99 \mathrm{MJ} \mathrm{m}^{-3} \mathrm{~K}^{-1}$ for silica, $(\rho c)_{\mathrm{g}}=2.51 \mathrm{MJ} \mathrm{m}^{-3} \mathrm{~K}^{-1}$ for gold, and $k_{\mathrm{t}}=0.445 \mathrm{~W} \mathrm{~m}^{-1} \mathrm{~K}^{-1}$ for dermis [15], one can obtain $t_{\mathrm{c}} \approx 2$ ns. This estimate shows that thermal nonequilibrium of the gold nanoshells and the surrounding tissue can be neglected for at least $\mu$ s-long radiation pulses. For short-pulsed laser radiation with pulse width $t_{\mathrm{p}} \ll 2 \mathrm{~ns}$, the possible overheating of GNPs during the laser pulse should be taken into account.

When GNPs are exposed to a short-pulsed laser radiation, the temperature of gold nanoshells will rise significantly and much faster (estimated heating time is about 2 ps [59]) than that of adjacent surrounding tissue (estimated heating time is about $100 \mathrm{ps}$ [59]). This is due to the ultrafast dynamics of photon-to-electron and electron-to-phonon energy conversion processes in metal nanoparticles [60]. Based on the heating times, the gold nanoshells will have considerably greater temperature than that of ambient tissue during a few nanoseconds after the pulse. In the microscale thermal model, the absorbed power by the gold shell of a nanoparticle and that by ambient tissue in each computational cell have to be determined. For a monochromatic incident radiation, the volumetric absorbed power by GNPs located within the computational cell $i_{z}$ and at time point $i_{t}$, can be directly determined from the quantity $N_{\mathrm{a}}\left(i_{t}, i_{r}, i_{z}\right)$ by:

$W_{\mathrm{p}}(t, r, z) \approx\left(1-\rho_{\mathrm{n}}\right) w \frac{\Psi\left(i_{r}, i_{z}\right) N_{\mathrm{a}}\left(i_{t}, i_{r}, i_{z}\right)}{\Delta t V_{\mathrm{g}}\left(i_{r}, i_{z}\right)}$

$\Psi\left(i_{r}, i_{z}\right)=\alpha_{\mathrm{p}} /\left(\alpha_{\mathrm{p}}+\alpha_{\mathrm{t}}\right) \quad V_{\mathrm{g}}\left(i_{\mathrm{r}}, i_{z}\right)=\left(1-\delta^{3}\right) f_{\mathrm{v}} V\left(i_{r}, i_{z}\right)$

where $\Psi\left(i_{r}, i_{z}\right)$ is the probability for a ray to be absorbed by a GNP located at the radius $r$ and abscissa $z$. $V_{\mathrm{g}}\left(i_{r}, i_{z}\right)$ is the volume of gold shells contained within the cell of indexes $i_{r}$ and $i_{z}$.

\subsection{Time-averaged absorbed power}

It is convenient to introduce time-averaged absorbed powers, which indicate if the quasi-steady approach is applicable for calculations of the absorbed radiation power for the case of pulsed irradiation of duration $\tau$. The time-averaged absorbed power for use in macroscopic thermal equilibrium model can be obtained as follows:

$\bar{W}^{*}\left(\tau_{\mathrm{m}}, r, z\right)=\frac{1}{\tau_{\mathrm{m}}} \int_{0}^{\tau_{\mathrm{m}}} W^{*}(t, r, z) d t \quad \tau_{\mathrm{m}} \geqslant \tau$

with $\tau_{\mathrm{m}}$ is the period used to compute the time-averaged absorbed powers. For each pulse width, the value of $\tau_{\mathrm{m}}$ should be adjusted to determine when the quasi-steady approach is applicable.

\section{Computational results}

The optical properties of three superficial human tissues of total thickness $d=3.5 \mathrm{~mm}$ at vacuum wavelength $\lambda=0.6328 \mu \mathrm{m}$ are specified in Table 1 . These data are taken from paper [9].

\subsection{Quasi-steady solution for absorbed radiation power}

The absorbed radiation power results obtained from the 1-D modified two-flux approximation [19] at various volume fractions of gold nanoshells are presented in Fig. 3. The external radiation is absorbed mainly in a surface layer of the irradiated medium and the absorbed specific power decreases with the distance from the illuminated surface. A local increase of volumetric absorption in the GNPs suspension is observed at $z=0.1 \mathrm{~mm}$. The referenced numerical solutions along the laser beam axis at $r=r_{c}=\left(r_{1}+r_{2}\right) / 2$ obtained using the quasi-steady MC method at $f_{\mathrm{v}}=0$ and $f_{\mathrm{v}}=2$ $\times 10^{-6}$ are also shown in Fig. 3. A relative difference between two methods increases in the region of a very low absorption (at $z>2 \mathrm{~mm}$ ). At the same time, one can see good agreement between approximate 1-D and exact solutions in the quasi-steady approach at least in the region of $z<2 \mathrm{~mm}$. We will focus on the results for this important region in subsequent unsteady calculations.

\subsection{Transient solution for absorbed radiation power}

The variation of the time-averaged absorbed power along the laser beam axis at $r=r_{c}$ for different pulse widths $t_{\mathrm{p}}$ obtained using transient Monte Carlo algorithm is analyzed below. The transient results are also compared with the results from traditional quasi-steady approach. Figs. 4a and b present the results for tissues with and without GNPs, respectively. The time-averaged results are calculated over period equal to the pulse duration, i.e. for $\tau_{m}=\tau$ in Eq. (19). Both a single laser pulse and a train of 4 pulses were considered. It can be seen that for very short pulses $\left(t_{\mathrm{p}} \leqslant 3 \mathrm{ps}\right)$, the time-averaged absorbed power has a significantly 
Table 1

Optical properties of tissues in the computational region.

\begin{tabular}{lllll}
\hline Layer number & Tissue name & Layer thickness $(\mathrm{mm})$ & $\alpha_{\mathrm{t}}\left(\mathrm{mm}^{-1}\right)$ & $\sigma_{\mathrm{tr}, \mathrm{t}}\left(\mathrm{mm}^{-1}\right)$ \\
\hline 1 & Epidermis & 0.1 & 0.30 & 2.50 \\
2 & Dermis & 1.4 & 0.27 & 3.75 \\
3 & Fat & 2.0 & 0.19 & 2.70 \\
\hline
\end{tabular}

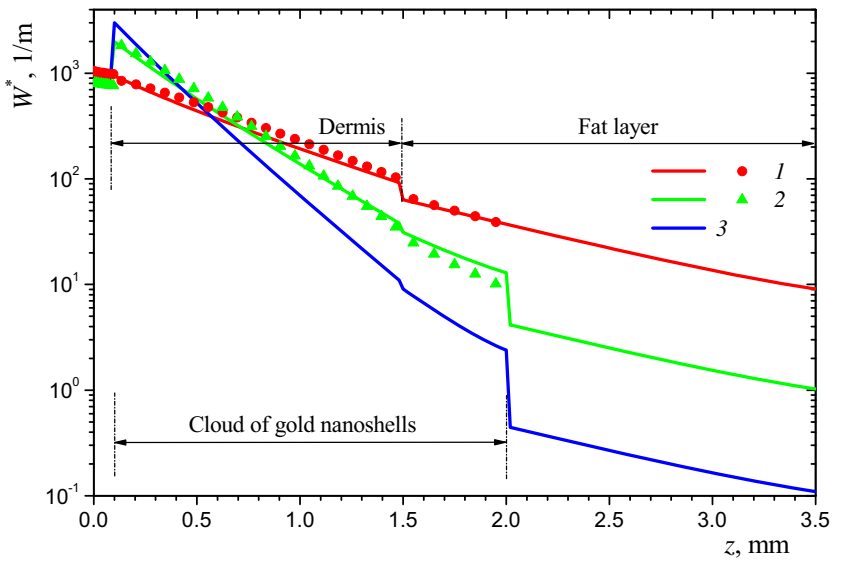

Fig. 3. Profiles of the normalized absorbed radiation power obtained using the quasi-steady approach: $1-f_{\mathrm{v}}=0,2-2 \times 10^{-6}, 3-5 \times 10^{-6}$. Curves - the modified two-flux approximation, symbols - the MCRT results at $r=r_{\mathrm{c}}$.

large value only in a relatively small depth of the tissue $(z<0.5 \mathrm{~mm})$. Radiation cannot penetrate further into the tissue during the time interval $0-\tau$ because of intense scattering. This result is in good qualitative agreement with the computational results of the recent study [49]. Thus, the quasi-steady approach is not applicable for very short pulses. For the pulses with $t_{\mathrm{p}}>10-$ ps, the differences between the quasi-steady and time-averaged results decrease. In these cases, the pulse durations ( $\tau>60$ ps for single pulses here) are relatively long so that radiation can propagate further into the tissue $(z>2 \mathrm{~mm})$. For the case of single pulse of width $t_{\mathrm{p}}=30 \mathrm{ps}$ and train of pulses of width $t_{\mathrm{p}}=10 \mathrm{ps}$, the results from the quasi-steady and transient approaches are very close to each other. That means that in these cases the quasi-steady approach is applicable to calculate the absorbed power for the macroscopic thermal problem with and without GNPs

Fig. 5 illustrates the change in the value of $\tau_{\mathrm{m}}$ resulting in a relative deviation less than $5 \%$ between the quasi-steady predictions and the time-averaged results for different pulses widths. This result can be interpreted as follows. If the characteristic time for heat conduction is larger than $\tau_{\mathrm{m}}$, the quasi-steady approach to radiative transfer is applicable; otherwise, the transient approach should be used to determine the absorbed powers. For the particular case of a train of pulses, $\tau_{\mathrm{m}}$ can be interpreted as the minimal time separating successive pulses, i.e. the pulse period, for which the quasi-steady approach is applicable.

\subsection{Radiation power absorbed by a single gold nanoparticle}

The transient radiation power absorbed by GNPs and by the surrounding tissue can be considered using the microscopic thermal non-equilibrium approach. In this section, we focus on the radiation power absorbed by a single GNP. It is especially important at the first moment of short radiation pulses when the irradiation is mainly absorbed by the gold shells of the GNPs through
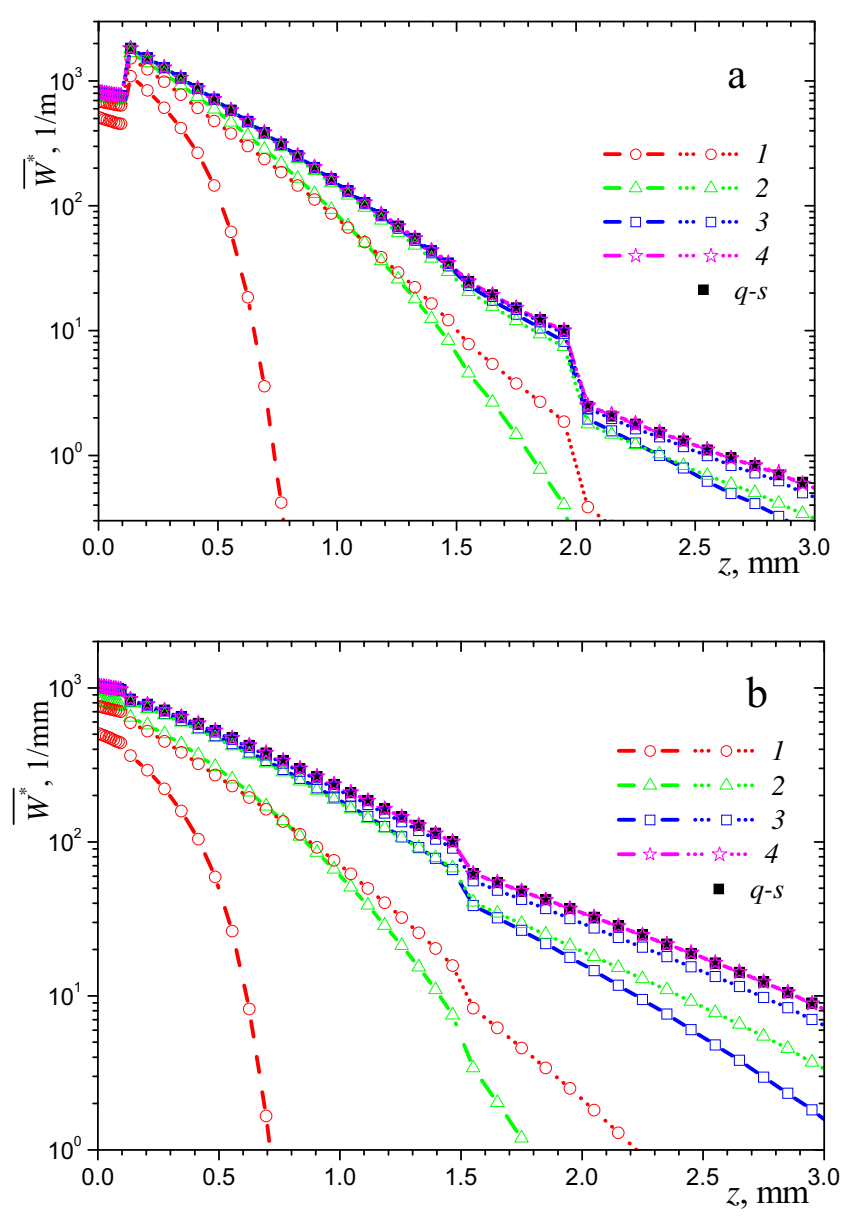

Fig. 4. Profiles of normalized absorbed radiation power for a single pulse (dashed curves with symbols) and a train of four pulses (dotted curves with symbols) for the cases (a) with GNPs and (b) without GNPs: $1-t_{\mathrm{p}}=1 \mathrm{ps}, 2-3 \mathrm{ps}, 3-10 \mathrm{ps}, 4-30 \mathrm{ps}, q-$ $s$ - quasi-steady solution.

photon-electron interactions. Through electron-phonon interactions, the absorbed energy results in heating the gold nanoshells during about 2 ps [59] and then the stored thermal energy in the GNP is transferred toward the surrounding dermis by heat conduction.

The maximal overheating for a given pulsed laser of energy density $P(\tau)$ can be estimated from energy conservation by assuming that there is no heat conduction toward the ambient dermis:

$\Delta T_{p}(t, r, z)=\frac{\int_{0}^{t} W_{\mathrm{p}}\left(t^{\prime}, r, z\right) d t^{\prime}}{(\rho c)_{\mathrm{p}}} \quad t \ll t_{c}$

where $W_{\mathrm{p}}$ the volumetric power absorbed by GNPs. Eq. (20) is applicable for pulse duration much shorter than the GNP cooling time $\left(\tau \ll t_{\mathrm{c}}\right.$ ). For longer pulse durations, $\tau>t_{\mathrm{c}}$, the total energy of the pulse does not convert entirely to the heating of the GNP due to the heat losses toward the surrounding medium. An estimate of 


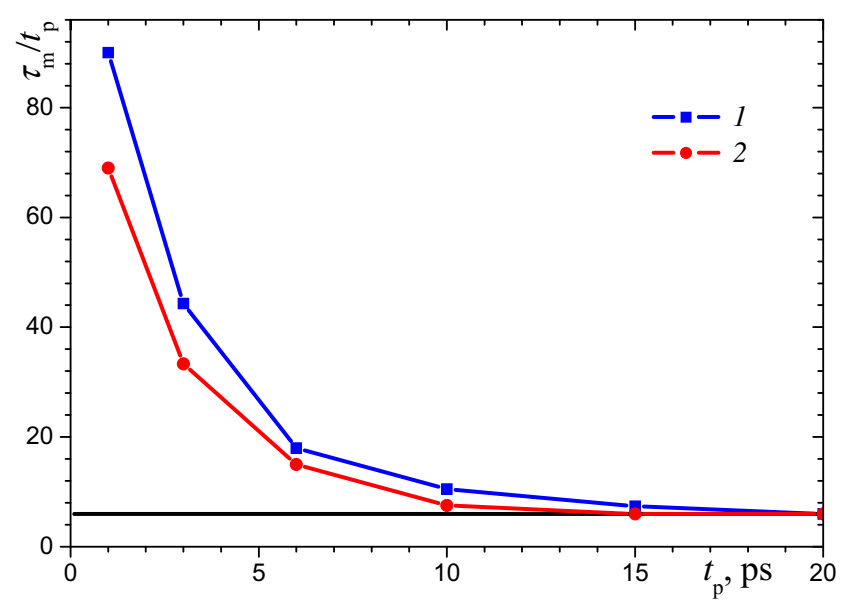

Fig. 5. Value of $\tau_{\mathrm{m}}$ corresponding to a relative deviation less than $5 \%$ of quasisteady absorbed powers with respect to the time-averaged absorbed powers: 1 without GNPs; 2 - with GNPs. Black line refers to the dimensionless single pulse duration $\tau_{\mathrm{m}} / t_{\mathrm{p}}=6$.

GNP's overheating accounting for such heat loss can be obtained from the solution of the classical transient energy equations in the particle and concentric dermis layer, surrounding the GNP:

$(\rho c)_{\mathrm{p}} \frac{\partial T}{\partial t}=\frac{k_{p}}{r^{\prime 2}} \frac{\partial}{\partial r^{\prime}}\left(r^{\prime 2} \frac{\partial T}{\partial r^{\prime}}\right)+W_{\mathrm{p}}\left(t, r^{\prime}, z\right) \quad r^{\prime}<a$

$(\rho c)_{\mathrm{t}} \frac{\partial T}{\partial t}=\frac{k_{\mathrm{t}}}{r^{\prime 2}} \frac{\partial}{\partial r^{\prime}}\left(r^{\prime 2} \frac{\partial T}{\partial r^{\prime}}\right)+W_{\mathrm{t}}\left(t, r^{\prime}, z\right) \quad r^{\prime}>a$

$k_{\mathrm{p}} \frac{\partial T}{\partial r^{\prime}}=\left.k_{\mathrm{t}} \frac{\partial T}{\partial r^{\prime}} \quad T\right|_{a-0}=\left.T\right|_{a+0} \quad r^{\prime}=a$

$T_{t}(t, r, z)=T_{t}(0, r, z)+\int_{0}^{t} W_{\mathrm{t}}\left(t^{\prime}, r, z\right) d t^{\prime} /(\rho c)_{\mathrm{t}}$

$r^{\prime}=d_{\mathrm{pp}}, \quad t<d_{\mathrm{pp}}^{2} k_{\mathrm{t}} /(\rho c)_{\mathrm{t}}$

where $W_{\mathrm{t}}$ the volumetric power absorbed in dermis at the radius $r$ and space coordinate $z$ of the computational domain; $r$ denotes the radial axis of the nanoparticle (it should not be confused with $r$, the radius of the computational domain); $d_{p p}=4 a /\left(3 f_{\mathrm{v}}\right)$ is the distance between neighboring nanoparticles. It is important to note that although the Fourier based model (21) has been often used in literature to solve ultrafast transient problems (e.g. in [59-61]), is only an approximation as it neglects the transfer of thermal energy at the finite speed. The boundary condition (21d) is deduced from (21b) by neglecting of $\partial T / \partial r^{\prime}$ at large distances from the nanoparticles. For simplicity, we also neglect the possible thermal resistance at the gold nanoshell/dermis interface, and the heating from the neighboring GNPs. The first assumption seems to be realistic in the case where there is no explosive evaporation of water from the surrounding dermis. The heating due to the neighboring GNPs can be neglected for short heating duration because it is known to be significant only for a time scale of approximately $d_{\mathrm{pp}}^{2} k_{\mathrm{t}} /(\rho c)_{\mathrm{t}} \sim 10^{-3} \mathrm{~s}[61]$. Due to the very small size of GNPs, the gold nanoshells are assumed to be isothermal. This last simplification enables us to recombine Eqs. (21a) and (21c), which leads to:

$(\rho c)_{\mathrm{p}} \frac{\partial T}{\partial t}-W_{\mathrm{p}}(t, r, z)=\frac{3}{r^{\prime}} k_{\mathrm{t}} \frac{\partial T}{\partial \mathrm{r}^{\prime}} \quad r^{\prime}=a$

Solution of Eq. (21b) taking into account Eqs. (21d) and (22) gives the temperature of an isothermal GNP. It can be used to determine the threshold incident radiation power, which may lead to explosive evaporation of water from the surrounding dermis. However, it is assumed in the present study that this threshold is not reached and the ordinary optical properties of gold nanoshells embedded in a human tissue [15] can be used.
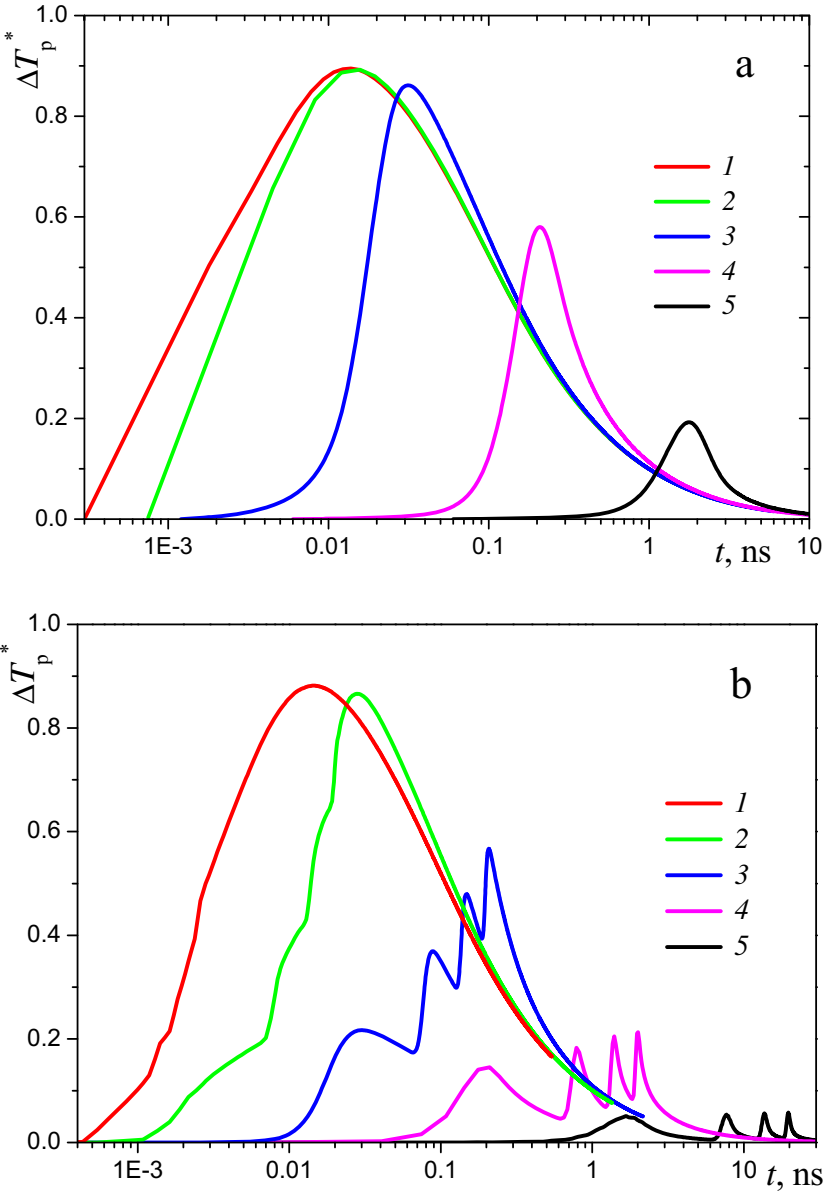

Fig. 6. Normalized time variation of a GNP overheating in response to (a) single laser pulse and (b) train of four laser pulses of $P(\tau)=1 \mathrm{pJ} \mu \mathrm{m}^{-2}$ energy density: $1-t_{\mathrm{p}}=0.1 \mathrm{ps}, 2-1 \mathrm{ps}, 3-10 \mathrm{ps}, 4-100 \mathrm{ps}, 5-1 \mathrm{~ns}$.

Fig. 6 shows the transient evolution of the overheating excess temperature of a single GNP, $\Delta T_{\mathrm{p}}(t, r, z)=T_{\mathrm{p}}(t, r, z)-T_{\mathrm{p}}(0, r, z)$, positioned in the dermis layer at $r=r_{\mathrm{c}}$ and $z=0.135 \mathrm{~mm}$ in response to a single laser pulse or a train of four laser pulses. Note that dermis layer is especially interesting for consideration of superficial tumors [15]. According to Fig. 4b, the absorbed power is maximal at this location and the overheating is expected to be the most severe. Five different pulse widths $t_{\mathrm{p}}$ ranging from $0.1 \mathrm{ps}$ to $1 \mathrm{~ns}$ are considered. In each case, the incident pulse energy flux was assumed equal to $P(\tau)=1 \mathrm{pJ} \mu \mathrm{m}^{-2}$, a typical value for picoseconds pulsed lasers. Indeed, identical irradiation energy allows us to investigate the effect of the pulse width on the maximum overheating temperature excess of GNPs. Note that in the absence of heat transfer toward the dermis, Eq. (20) gives $\Delta T_{\mathrm{p}}=77 \mathrm{~K}$. This value was used to normalize the results presented in Fig. 6.

In both cases of single pulse and train of four pulses, the narrower the pulse width, the higher the nanoparticle overheating. It is clear from these figures that very short laser pulses allow to achieve a significant overheating during a long time period. On the contrary, long pulses lead to moderate and small overheating. The computational results for the case of short-pulsed irradiation with a single pulse are qualitatively similar to that with a train of four pulses $\left(t_{\mathrm{p}}=0.1\right.$ and $\left.1 \mathrm{ps}\right)$. The largest value of the overheating excess temperature, obtained with laser of pulse width $t_{\mathrm{p}}=0.1 \mathrm{ps}$, is about $69 \mathrm{~K}$, inferior to the limit value $77 \mathrm{~K}$ due to significant heat conduction to dermis even at very short pulses. 


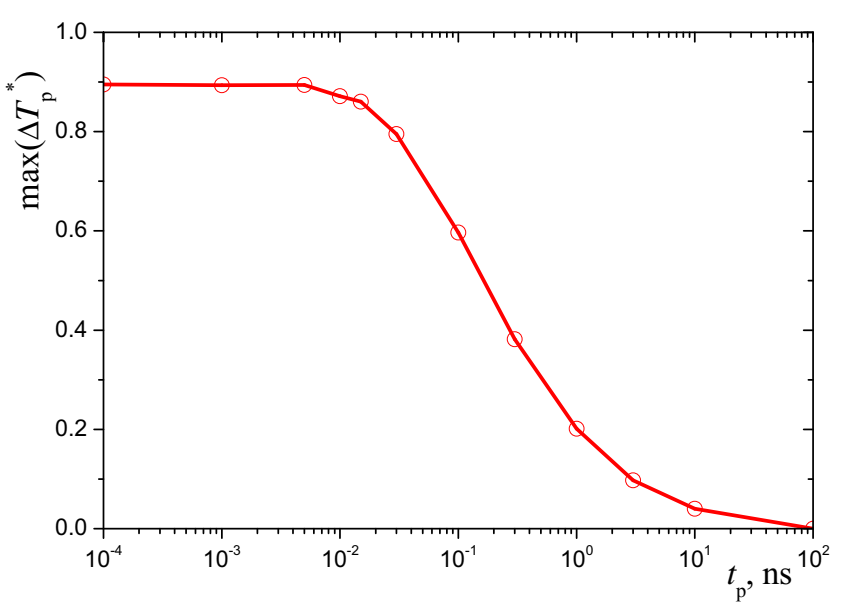

Fig. 7. Variation of normalized maximum of GNP overheating with the pulse width in the case of single laser pulse of $P(\tau)=1 \mathrm{pJ} \mu \mathrm{m}^{-2}$ energy density.

Therefore, it is important to model the entire heat transfer problem when evaluating GNP's temperature.

Fig. 7 presents a variation of the overheating excess temperature maxima as a function of the pulse width for the case of a single pulse. For pulse widths $t_{\mathrm{p}}<10 \mathrm{ps}$, the overheating is large and it is weakly dependent on $t_{\mathrm{p}}$. In the case of $t_{\mathrm{p}}>10 \mathrm{ps}$, the maximum overheating decreases abruptly when the pulse width whereas the overheating is very small at $t_{\mathrm{p}}>0.1 \mu$ s (less than $1 \mathrm{~K}$ for the problem under consideration). The data of Fig. 7 can be used to choose the pulsed laser parameters for thermal treatment. The latter is especially important for the direct laser damage [6] of tumor cells with embedded GNPs.

\subsection{Accuracy considerations}

In the MCRT method, the transient absorbed radiative power is obtained as an average result of ten independent runs. Each run consists of tracking $5 \times 10^{7}$ rays and uses a unique seed for the random number generator. The computational time of a single run is about $5 \mathrm{~min}$ on a desktop computer Intel(R) Core(TM) i3-2330 M CPU@ $2.2 \mathrm{GHz}$ processor with $4 \mathrm{~GB}$ of RAM. The relative error is estimated by comparing the transient absorbed power obtained for $N_{\text {ray }}=10^{7}, 5 \times 10^{7}$ and $5 \times 10^{8}$. The accuracy of the numerical solution is quantified by computing a standard deviation based on the results of ten runs, each with $N_{\text {ray }}$ rays and a different value of the random number generator seed. Typical axial distributions of this relative error for a 1 ps pulse of laser radiation are presented in Fig. 8. Only the important part of the computational region characterized by a considerable value of the absorbed power is shown in this figure. In all cases, the relative error is less than $1 \%$ in the region of $z<2 \mathrm{~mm}$, where the number of rays is sufficient to obtain accurate results. A non-uniform computational mesh used to obtain reliable numerical results was discussed in paper [19].

The effects of uncertainties in the input parameters on the computational results are analyzed at three typical pulse widths, $3 \mathrm{ps}$, $10 \mathrm{ps}$ and $1 \mathrm{~ns}$. Simulation results for tissues with and without GNPs are assessed. The uncertainties of $10 \%$ in each of the input parameters are considered. It is shown that the uncertainty in the tissue refractive index results in less than $6 \%$ change in absorbed radiation power and $3.5 \%$ in the dermis temperature. The uncertainty in transport scattering coefficient leads to less than $2 \%$ deviation in the absorbed radiation power and less than $3 \%$ in temperature of tissues. The same uncertainty in absorption coefficient is more important, at least for the case without GNPs. It leads to almost $9 \%$ variation in the absorbed radiation power.

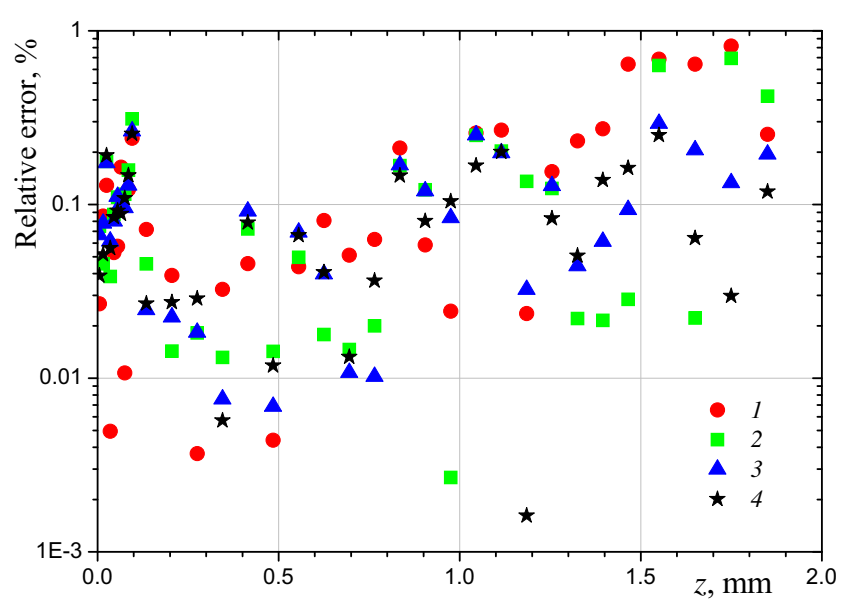

Fig. 8. Relative error in time-averaged absorbed radiation power for laser pulse width of 1 ps: $1-\tau_{m}=14 \mathrm{ps}, 2-22 \mathrm{ps}, 3-30 \mathrm{ps}, 4-200 \mathrm{ps}$.

Table 2

Thermal properties of tissues in the computational region.

\begin{tabular}{lllll}
\hline Layer number & Tissue name & $\rho_{\mathrm{t}}\left(\mathrm{kg} \mathrm{m}^{-3}\right)$ & $c_{\mathrm{t}}\left(\mathrm{J} \mathrm{kg}^{-1} \mathrm{~K}^{-1}\right)$ & $k_{\mathrm{t}}\left(\mathrm{W} \mathrm{m}^{-1} \mathrm{~K}^{-1}\right)$ \\
\hline 1 & Epidermis & 1200 & 3589 & 0.235 \\
2 & Dermis & 1200 & 3300 & 0.445 \\
3 & Fat & 1000 & 2674 & 0.185 \\
\hline
\end{tabular}

As one can expect, the effect of uncertainties in thicknesses of tissue layers is negligible (less than 1\%). Finally, the uncertainties in thermophysical properties of tissues affect considerably (up to $6 \%$ ) the calculated temperature in the case of long pulse duration.

\section{Effect of short-pulsed radiation on macroscale heating of superficial human tissues}

In hyperthermia, the temperature variation due to thermal treatment is usually much greater than the difference between local temperatures of the tissue and blood. Therefore, this difference can be neglected, at least in the approximate thermal model. In subsequent analysis we neglect also convective heat transfer with arterial blood. The latter is an acceptable approach for superficial human tissues [9]. According to computational results presented in [9], we use a one-temperature model assuming the local thermal equilibrium between arterial blood and ambient tissues. A small value of the metabolic heat generation in superficial tissues is neglected. In this case, the 1-D energy equation and the associated initial and boundary conditions are:

$(\rho c)_{\mathrm{t}} \frac{\partial T_{\mathrm{t}}}{\partial t}=\frac{\partial}{\partial z}\left(k_{\mathrm{t}} \frac{\partial T_{\mathrm{t}}}{\partial z}\right)+q_{\mathrm{inc}} \bar{W}^{*}(z)$

$t=0 \quad T_{\mathrm{t}}=T_{\mathrm{t}, \mathrm{i}}(z)$

$z=0 \quad k_{\mathrm{t}} \frac{\partial T_{\mathrm{t}}}{\partial z}=h_{1}\left(T_{\mathrm{e}, 1}-T_{\mathrm{t}}\right) \quad z=d \quad k_{\mathrm{t}} \frac{\partial T_{\mathrm{t}}}{\partial z}=h_{2}\left(T_{\mathrm{e}, 2}-T_{\mathrm{t}}\right)$

where $q_{\text {inc }}$ is the averaged incident radiative flux. It is assumed that $q_{\text {inc }}=$ const during the irradiation. The boundary condition at $z=0$ corresponds to cooling of the body surface by a mixed (forced and natural) convection, whereas the boundary condition at $z=d$ (at the boundary between fat and muscle layers) describes approximately the heat transfer from the internal part of the body. The initial steady-state temperature profile $T_{\mathrm{t}, \mathrm{i}}(z)$ is a solution to the following boundary-value problem:

$\frac{d}{d z}\left(k_{t} \frac{d T_{t}}{d z}\right)=0$ 
Table 3

Heat transfer parameters.

\begin{tabular}{lllll}
\hline$h_{1}\left(\mathrm{~W} \mathrm{~m}^{-1} \mathrm{~K}^{-1}\right)$ & $h_{2}\left(\mathrm{~W} \mathrm{~m}^{-1} \mathrm{~K}^{-1}\right)$ & $T_{\mathrm{e}, 1}(\mathrm{~K})$ & $T_{\mathrm{e}, 2}(\mathrm{~K})$ & $\begin{array}{l}q_{\text {inc }}\left(\mathrm{W} \mathrm{m}^{-2}\right) \text { with } \\
\text { GNRs/without GNPs }\end{array}$ \\
\hline 10 & 50 & 295 & 310 & $1500 / 1900$
\end{tabular}

$z=0 \quad k_{\mathrm{t}} \frac{d T_{\mathrm{t}}}{d z}=h_{1}\left(T_{\mathrm{e}, 1}-T_{\mathrm{t}}\right) \quad z=d \quad k_{\mathrm{t}} \frac{d T_{\mathrm{t}}}{d z}=h_{2}\left(T_{\mathrm{e}, 2}-T_{\mathrm{t}}\right)$

The main parameters of the above heat transfer problem are specified in Tables 2 and 3 [9,15].

The computational results in the form of temperature profiles at different time moments are presented in Fig. 9. It can be seen that temperature of human tissues in the case of a short-pulse irradiation is systematically less that that predicted by the quasi-steady solution for radiative transfer. This difference is especially great when there are no GNPs in the body (Fig. 9b). As was mentioned above, this effect is explained by a relatively large reflection of the short pulsed laser radiation by highly scattering superficial human tissues. At the same time, the temperature profiles appeared to be very close to the quasi-steady solution in the case of pulse width greater than 10 ps. This effect is much stronger in the case when GNPs are embedded in human tissues (Fig. 9a): the temperature curves $T_{\mathrm{t}}(z)$ at $t_{\mathrm{p}}=10 \mathrm{ps}$ are practically indistinguishable of similar curves calculated using relatively simple quasi-steady approach.
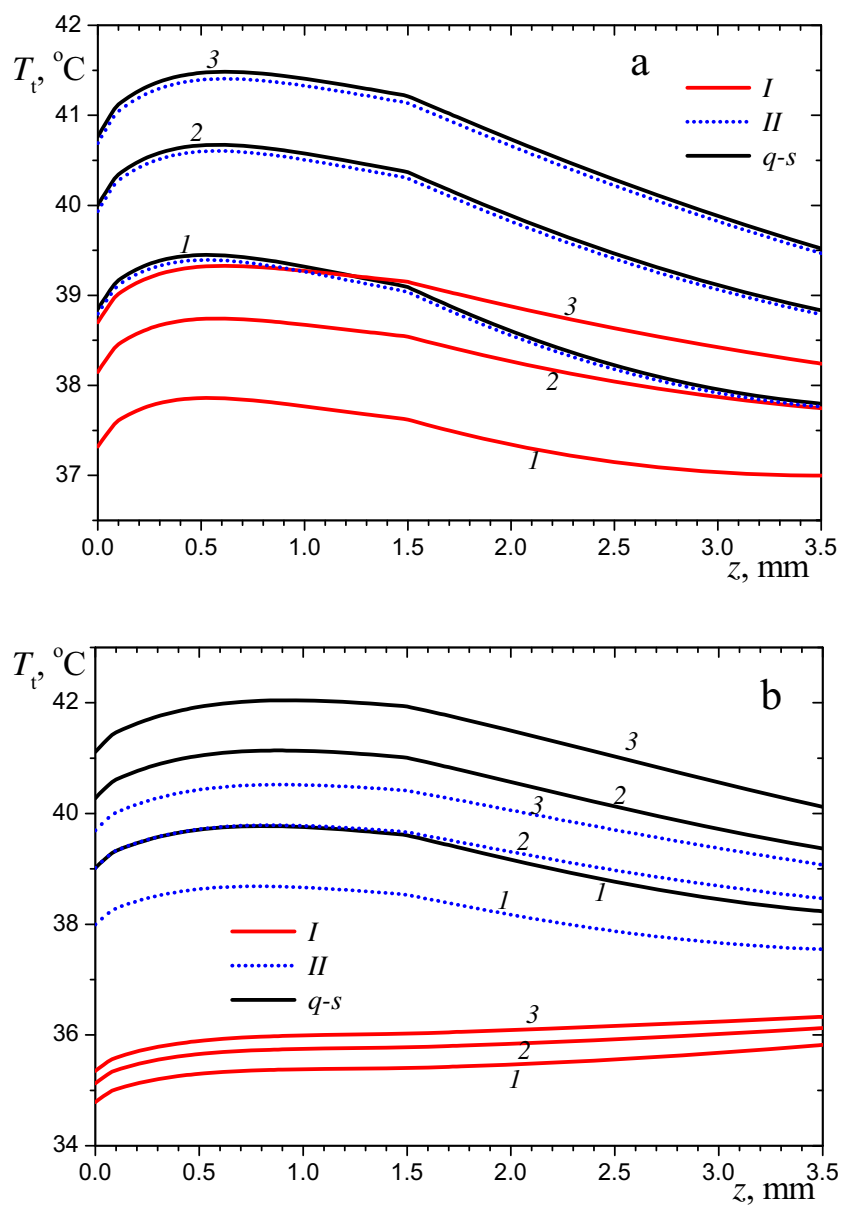

Fig. 9. Temperature profiles in human tissues at different time moments: $1-t=2 \mathrm{~s}, 2-3 \mathrm{~s}, 3-4 \mathrm{~s}$. Calculations for single-pulsed irradiation (a) with GNPs and (b) without GNPs: I $-t_{\mathrm{p}}=3 \mathrm{ps}$, II-10 ps, $q-s-$ quasi-steady solution.

\section{Conclusions}

The Monte Carlo method has been employed to analyze the effects of finite speed of light during the interaction of short-pulsed laser radiation with human tissues as applied to thermal treatment of superficial tumors. The increase of the local radiation absorption in the tissue in the therapeutic window was examined for the short-pulsed radiation with gold nanoparticles (GNPs) embedded in human tissues.

It was shown that the quasi-steady approach neglecting the final speed of light is applicable for human tissues without GNPs for laser pulse width greater than $10 \mathrm{ps}$. At the same time, the microscale heat transfer in the vicinity of single GNPs is more sensitive to the duration of laser pulses because of the considerable overheating effect of the particles with respect to ambient tissue. The thermal nonequilibrium is pronounced at pulse duration less than about 1 ns but the steady-state approach remains to be applicable to estimate the overheating at laser pulse width greater than $10 \mathrm{ps}$. In the case of shorter pulses, the effect of finite speed of light should be taken into account in solving microscale problems. The latter is expected to be especially important in the case of a direct irradiation of superficial tumors with embedded nanoparticles.

A heat transfer problem for multilayer superficial human tissues in the case of the external short-pulse laser irradiation was solved on the basis of the Fourier law and various approaches for the average absorption of the incident radiation. It was shown that temperature of the tissues in the case of a short-pulse irradiation is systematically less that that predicted by the quasi-steady solution for radiative transfer. This difference is especially large when there are no GNPs in the body (Fig. 9b). This effect can be explained by a relatively strong reflection of the short pulsed laser radiation by highly scattering superficial human tissues. At the same time, the temperature profiles were closer to those obtained using the quasi-steady solution in the case of pulse width greater than $10 \mathrm{ps}$.

\section{Conflict of interest}

None declared

\section{Acknowledgment}

Leonid Dombrovsky is grateful for the partial financial support of this study from the Russian Foundation for Basic Research (Grant no. 13-08-00022a).

\section{References}

[1] J. Jiao, Z. Guo, Thermal interaction of short-pulsed laser focused beams with skin tissues, Phys. Med. Biol. 54 (13) (2009) 4225-4241.

[2] X. Huang, B. Kang, W. Qian, M.A. Mackey, P.C. Chen, A.K. Oyelere, I.H. El-Sayed, M.A. El-Sayed, Comparative study of photothermolysis of cancer cells with nuclear-targeted or cytoplasm-targeted gold nanospheres: continuous wave or pulsed lasers, J. Biomed. Opt. 15 (5) (2010) 058002.

[3] A.Y. Sajjadi, K. Mitra, M. Grace, Ablation of subsurface tumors using an ultrashort pulse laser, Opt. Lasers Eng. 49 (3) (2011) 451-456.

[4] J. Mobley, T. Vo-Dinh, Optical properties of tissue, in: T. Vo-Dinh (Ed.), Biomedical Photonics Handbook, CRC Press, Boca Raton, FL, 2003, pp. 2-1-275.

[5] V.V. Tuchin, Tissue Optics: Light Scattering Methods and Instruments for Medical Diagnosis, vol. PM166, SPIE Press, Bellingham, WA, 2007. second ed.

[6] M.H. Niemz, Laser-tissue interactions: fundamentals and applications, in: Biological and Medical Physics Series, second ed., Springer, Berlin, 2002.

[7] J. Vera, Y. Bayazitoglu, Gold nanoshell density variation with laser power for induced hyperthermia, Int. J. Heat Mass Transfer 52 (3-4) (2009) 564-573.

[8] X. Huang, M.A. El-Sayed, Gold nanoparticles: optical properties and implementations in cancer diagnosis and photothermal therapy, J. Adv. Res. 1 (1) (2010) 13-28.

[9] L.A. Dombrovsky, V. Timchenko, M. Jackson, Indirect heating strategy of laser induced hyperthermia: an advanced thermal model, Int. J. Heat Mass Transfer 55 (17-18) (2012) 4688-4700. 
[10] V. Timchenko, L. Dombrovsky, Laser induced hyperthermia of superficial tumors: a transient thermal model for indirect heating strategy, Comput. Therm. Sci. 4 (6) (2012) 457-475.

[11] N.G. Khlebtzov, L.A. Dykman, Optical properties and biomedical applications of plasmonic nanoparticles, J. Quant. Spectrosc. Radiat. Transfer 111 (1) (2010) $1-35$.

[12] B.N. Khlebtsov, V.A. Khanadeev, E.P. Panfilova, T.E. Pylaev, O.A. Bibikova, S.A. Staroverov, V.A. Bogatyrev, L.A. Dykman, N.G. Khlebtsov, New types of nanomaterials: powders of gold nanospheres, nanorods, nanostars, ad goldsilver nanocages, Nanotech. Russia 8 (3-4) (2013) 209-219.

[13] Y. Bayazitoglu, S. Kheradmand, T.K. Tullius, An overview of nanoparticle assisted laser therapy, Int. J. Heat Mass Transfer 67 (2013) 469-486.

[14] G. Terentyuk, E. Panfilova, V. Khanadeev, D. Chumakov, E. Genina, A. Bashkatov, V. Tuchin, A. Bucharskaya, G. Maslyakova, N. Khlebtsov, B. Khlebtsov, Gold nanorods with a hematoporphyrin-loaded silica shell for dual-modality photodynamic and photothermal treatment of tumors in vivo, Nano Res. 7 (3) (2014) 325-337.

[15] L.A. Dombrovsky, V. Timchenko, M. Jackson, G.H. Yeoh, A combined transient thermal model for laser hyperthermia of tumors with embedded gold nanoshells, Int. J. Heat Mass Transfer 54 (25-26) (2011) 5459-5469.

[16] L.A. Dombrovsky, J.H. Randrianalisoa, W. Lipiński, V. Timchenko, Simplified approaches to radiative transfer simulations in laser induced hyperthermia of superficial tumors, Comput. Therm. Sci. 5 (6) (2013) 521-530.

[17] L.A. Dombrovsky, D. Baillis, Thermal Radiation in Disperse Systems: An Engineering Approach, Begell House, New York, 2010.

[18] L.A. Dombrovsky, The use of transport approximation and diffusion-based models in radiative transfer calculations, Comput. Therm. Sci. 4 (4) (2012) 297-315.

[19] L. Dombrovsky, J. Randrianalisoa, D. Baillis, Modified two-flux approximation for identification of radiative properties of absorbing and scattering media from directional-hemispherical measurements, J. Opt. Soc. Am. A 23 (1) (2006) 91-98.

[20] L.H. Liu, H.P. Tan, T.W. Tong, Non-Fourier effects on transient temperature response in semitransparent medium caused by laser pulse, Int. J. Heat Mass Transfer 44 (17) (2001) 3335-3344.

[21] F. Xu, K.A. Seffen, T.J. Lu, Non-Fourier analysis of skin biothermomechanics, Int. J. Heat Mass Transfer 51 (9-10) (2008) 2237-2259.

[22] M.M. Najafabadi, A.S. Ghazani, H.B. Tabrizi, Numerical simulation of nonFourier temperature behavior of functionally graded materials in a slab with surface radiation, Numer. Heat Transfer A 63 (3) (2013) 226-243.

[23] T.J. Bright, Z.M. Zhang, Common misperceptions of the hyperbolic heat equation, AIAA J. Thermophys. Heat Transfer 23 (3) (2009) 601-607.

[24] T.J. Bright, G.P. Peterson, Reexamination of the statistical derivations of Fourier's law and Cattaneo's equation, Nanoscale Microscale Thermophys. Eng. 15 (4) (2011) 220-228.

[25] S.A. Rukolaine, Unphysical effects of the dual-phase-lag model of heat conduction, Int. J. Heat Mass Transfer 78 (2014) 58-63.

[26] D.Y. Tzou, Macro- to Microscale Heat Transfer: The Lagging Behavior, Taylor \& Francis, Washington DC, 1997.

[27] Z.M. Zhang, Nano/Microscale Heat Transfer, McGraw Hill, New York, 2007.

[28] B. Straughan, Heat Waves, Springer, New York, 2011.

[29] Z. Guo, S. Kumar, Equivalent isotropic scattering formulation for transient short-pulse radiative transfer in anisotropic scattering plane layer, Appl. Opt. 39 (24) (2000) 4411-4417.

[30] Z. Guo, S. Kumar, Radiation element method for hyperbolic radiative transfer in plane-parallel inhomogeneous media, Numer. Heat Transfer B 39 (4) (2001) 371-387.

[31] C.-J. Wu, N.-R. Ou, Differential approximation for transient radiative transfer through a participating medium exposed to collimated radiation, J. Quant. Spectrosc. Radiat. Transfer 73 (1) (2002) 111-120.

[32] Z. Guo, J. Aber, B.A. Garetz, S. Kumar, Monte Carlo simulation and experiments of pulsed radiative transfer, J. Quant. Spectrosc. Radiat. Transfer 73 (2-5) (2002) 159-168.

[33] R. Elaloufi, R. Carminati, J.-J. Greffet, Time-dependent transport through scattering media: from radiative transfer to diffusion, J. Optics A: Pure Appl. Opt. 4 (5) (2002) 103-108.

[34] K.H. Kim, Z. Guo, Ultrafast radiation heat transfer in laser tissue welding and soldering, Numer. Heat Transfer A 46 (1) (2004) 23-46.

[35] S.C. Mishra, P. Chung, P. Kumar, K., and Mitra, Development and comparison of the DTM, the DOM and the FVM formulations for the short-pulse laser transport through a participating medium, Int. J. Heat Mass Transfer 49 (1112) (2006) 1820-1832.

[36] P.S. Brantley, Angular adaptive $P_{1}$ - double $P_{0}$ flux-limited diffusion solutions of non-equilibrium grey radiative transfer problems, J. Quant. Spectrosc. Radiat. Transfer 104 (1) (2007) 116-132.

[37] M. Frank, A.S. Klar, E.W. Larsen, S. Yasuda, Time-dependent simplified $P_{\mathrm{N}}$ approximation to the equations of radiative transfer, J. Comput. Phys. 226 (2) (2007) 2289-2305

[38] T. Okutucu, Y. Yener, A.A. Busnaina, Transient radiative transfer in participating media with pulse-laser irradiation - an approximate Galerkin solution, J. Quant. Spectrosc. Radiat. Transfer 103 (1) (2007) 118-130.

[39] R. Singh, S.C. Mishra, N.K. Roy, N.S. Sekhawat, K. Mitra, An insight into the modeling of short-pulse laser transport through a participating medium, Numer. Heat Transfer B 52 (4) (2007) 373-385.

[40] R. Muthukumaran, S.C. Mishra, Interaction of a short-pulse laser of a Gaussian temporal profile with an inhomogeneous medium, Numer. Heat Transfer A 53 (6) (2008) 625-640.

[41] M.K. Jaunich, S. Raye, K. Kim, K. Mitra, Z. Guo, Bio-heat transfer analysis during short pulse laser irradiation of tissues, Int. J. Heat Mass Transfer 51 (23-24) (2008) 5511-5521.

[42] R. Muthukumaran, S.C. Mishra, Transient response of a planar participating medium subjected to train of short-pulse radiation, Int. J. Heat Mass Transfer $51(9-10)(2008) 2418-2432$.

[43] L.H. Liu, P.-F. Hsu, Time shift and superposition method for solving transient radiative transfer equation, J. Quant. Spectrosc. Radiat. Transfer 109 (7) (2008) 1297-1308.

[44] M. Bhuvaneswari, C.-Y. Wu, Differential approximations for transient radiative transfer in refractive planar medium with pulse irradiation, J. Quant. Spectrosc. Radiat. Transfer 110 (6-7) (2009) 389-401.

[45] L.M. Ruan, S.G. Wang, H. Qi, D.L. Wang, Analysis of the characteristics of timeresolved signals for transient radiative transfer in scattering participating media, J. Quant. Spectrosc. Radiat. Transfer 111 (16) (2010) 2405-2414.

[46] S.C. Mishra, R. Muthukumaran, S. Maruyama, Comparison of the thermal effects of the transport of a short-pulsed laser and multi-pulse laser through a participating medium, Int. J. Heat Mass Transfer 55 (21-22) (2012) 55835596.

[47] F. Asllanaj, S. Fumeron, Applying a new computational method for biological tissue optics based on the time-dependent two-dimensional radiative transfer equation, J. Biomed. Opt. 17 (7) (2012) 075007 (Erratum in Journal of Biomedical Optics 17 (7) (2012) 079801).

[48] Y. Zhang, H. Yi, H. Tan, One-dimensional transient radiative transfer by lattice Boltzmann method, Opt. Express 21 (21) (2013) 24532-24539.

[49] A. Bhowmik, R. Repaka, S.C. Mishra, K. Mitra, Analysis of radiative signals from normal and malignant human skins subjected to a short-pulse laser, Int. J. Heat Mass Transfer 68 (2014) 278-294.

[50] M. Born, E. Wolf, Principles of Optics, seventh expanded ed., Cambridge Univ. Press, New York, 1999.

[51] C.F. Bohren, D.R. Huffman, Absorption and Scattering of Light by Small Particles, Wiley, New York, 1983.

[52] S.L. Jacques, Optical properties of biological tissues: a review, Phys. Med. Biol. 58 (11) (2013) R37-R61.

[53] V.P. Scripov, Metastable Liquids, Wiley, New York, 1974.

[54] L.A. Dombrovsky, Radiation heat transfer from a hot particle to ambient water through the vapor layer, Int. J. Heat Mass Transfer 43 (13) (2000) 2405-2414.

[55] J.T. Farmer, J.R. Howell, Comparison of Monte Carlo strategies for radiative transfer in participating media, Adv. Heat Transfer 31 (1998) 333-429.

[56] J.R. Howell, R. Siegel, M.P. Mengüç, Thermal Radiation Heat Transfer, CRC Press, New York, 2010.

[57] M.F. Modest, Radiative Heat Transfer, Third ed., Acad. Press, New York, 2013.

[58] L.A. Dombrovsky, W. Lipiński, A combined $\mathrm{P}_{1}$ and Monte Carlo model for multi-dimensional radiative transfer problems in scattering media, Comput. Therm. Sci. 2 (6) (2010) 549-560.

[59] V.K. Pustovalov, Theoretical study of heating of spherical nanoparticle in media by short laser pulses, Chem. Phys. 308 (1-2) (2005) 103-108.

[60] G. Baffou, H. Rigneault, Femtosecond-pulsed optical heating of gold nanoparticles, Phys. Rev. B 84 (3) (2011) 035415.

[61] P. Keblinski, D.G. Cahill, A. Bodapati, C.R. Sillivan, T.A. Taton, Limits of localized heating by electromagnetically excited nanoparticles, J. Appl. Phys. 100 (5) (2006) 054305 